\title{
A CHOICE FUNCTION ON COUNTABLE SETS, FROM DETERMINACY
}

\author{
PAUL B. LARSON
}

(Communicated by Mirna Džamonja)

\begin{abstract}
We prove that $\mathrm{AD}_{\mathbb{R}}$ implies the existence of a definable class function which, given a countable set $X$, a tall ideal $I$ on $\omega$ containing Fin and a function from $I \backslash$ Fin to $X$ which is invariant under finite changes, selects a nonempty finite subset of $X$. Among other applications, this gives an alternate proof of the fact (previously established by Di Prisco-Todorcevic) that there is no selector for the $E_{0}$ degrees in the $\mathcal{P}(\omega) /$ Fin-extension of a model of $\mathrm{AD}_{\mathbb{R}}$.
\end{abstract}

An ideal $I$ on $\omega$ is said to be tall if every infinite subset of $\omega$ contains an infinite member of $I$. In this paper we show that, assuming the Axiom of Real Determinacy $\left(\mathrm{AD}_{\mathbb{R}}\right)$, there is a definable procedure which, given a tall ideal on $\omega$ containing Fin (the ideal of finite subsets of $\omega$ ) and a function on $I \backslash$ Fin which is invariant under finite changes and has countable range, selects a nonempty finite subset of the range of this function. In the case we are most interested in, $I$ is generated by a countable collection of pairwise orthogonal ideals. In this context, these ideals represent a $\mathcal{P}(\omega)$ /Fin-name for an element of a countable set from the ground model. Our result (Theorem 3.3) has the following consequence (Corollary [3.4): if

- $M[U]$ is a $\mathcal{P}(\omega) /$ Fin-extension of a model $M$ of $\mathrm{AD}_{\mathbb{R}}$,

- $F$ is a function in $M[U]$ with domain $Y \in M$,

- $G$ is a function in $M$ with domain $Y$ such that, for all $y \in Y, G(y)$ is countable and $F(y)$ is a finite nonempty subset of $G(y)$,

then there exists a function $G^{\prime}$ in $M$ with domain $Y$ such that, for all $y \in Y, G^{\prime}(y)$ is a nonempty finite subset of $G(y)$. This gives another proof of a theorem of Di Prisco-Todorcevic [2] (see also [6]) that there is no function in $M[U]$ which selects a single member from each $E_{0}$-equivalence class (where $E_{0}$ is the relation of a modfinite agreement on $\mathcal{P}(\omega)$ ). Since the finite sets of $E_{0}$ degrees can be injectively embedded into the (individual) $E_{0}$ degrees in a Borel fashion, this also implies that whenever $F$ is an equivalence relation on $\mathcal{P}(\omega)$ in $M$ whose equivalence classes are countable unions of $E_{0}$-classes, if a function in $M[U]$ picks an $E_{0}$ subclass for each $F$-class, then such a function exists already in $M$.

In the final section of the paper (Section 5) we note that "finite" cannot be replaced with "size $n$ " in our result, for any positive $n \in \omega$. In particular, since

Received by the editors March 28, 2013 and, in revised form, July 8, 2013 and September 2, 2013.

2010 Mathematics Subject Classification. Primary 03E25, 03E40, $03 E 60$.

This research was supported by NSF Grants DMS-0801009 and DMS-1201494. The results were obtained in February 2011. The author thanks Andrés Caicedo and Grigor Sargsyan for help with the bibliographic references. 
$\mathrm{AD}_{\mathbb{R}}$ implies that all sets of reals have the property of Baire, for any fixed positive $n \in \omega$ it is not possible in this context to pick $n$ members from each countable family of orthogonal ideals generating a tall ideal.

\section{StrategicAlly SELECTIVE IDEALS}

Given a sequence $\left\langle x_{n}: n \in \omega\right\rangle$ consisting of subsets of $\omega$, a one-point selector of this sequence is a set $\left\{b_{n}: n<\omega\right\}$, listed in increasing order, such that each $b_{n}$ is an element of the corresponding $x_{n}$. Given an ideal $I$ on $\omega$, we let $G(I)$ be the game where the players Noselector (who moves first) and Selector alternately pick the members of a $\subseteq$-decreasing sequence of $I$-positive sets, with Selector winning if the sequence produced has a one-point selector in $I^{+}$. We say that an ideal $I$ is strategically selective if Noselector does not have a winning strategy in $G(I)$. The proof of Theorem 1.1 below consists of combining work of Martin, Solovay and Woodin with Mathias's proof 4 that $\mathrm{AD}_{\mathbb{R}}$ implies that there are no tall selective ideals.

Theorem $1.1([3])$. If $\mathrm{AD}_{\mathbb{R}}$ holds, then there are no tall strategically selective ideals on $\omega$.

It follows that under $\mathrm{AD}_{\mathbb{R}}$, the strategically selective ideals are exactly those ideals $I$ such that every infinite $A \subseteq \omega$ contains an infinite $B \subseteq \omega$ with $\mathcal{P}(B) \cap I$ containing no infinite set.

For the definition of strategically selective ideals, it is appropriate for Noselector to move first, but for the purposes of this paper Selector moves first in the relevant game. We therefore let $G^{\prime}(I)$ be a modified game in which Selector moves first, and again the two players alternately pick the members of a $\subseteq$-decreasing sequence of $I$-positive sets, with Selector winning if the sequence produced has a selector in $I^{+}$. Theorem 1.1 implies that under $\mathrm{AD}_{\mathbb{R}}$, Noselector still has a winning strategy in this game whenever $I$ is a tall ideal on $\omega$.

Corollary 1.2. If $\mathrm{AD}_{\mathbb{R}}$ holds, then Noselector has a winning strategy for $G^{\prime}(I)$ whenever $I$ is a tall ideal on $\omega$.

\section{Embedding the Baire SPACE}

For each $\subseteq$-decreasing sequence $\bar{x}=\left\langle x_{n}: n \in \omega\right\rangle$ consisting of infinite subsets of $\omega$, we recursively define an association of finite sequences $\sigma \in \omega^{<\omega}$ to finite subsets $z_{\sigma}^{\bar{x}} \subseteq \omega$ as follows:

$$
\begin{aligned}
& \text { - } z_{\langle\rangle}^{\bar{x}}=\emptyset \text {; } \\
& \text { - } z_{\sigma}^{\bar{x}}\left\langle\langle 0\rangle=z_{\sigma}^{\bar{x}}\right. \text {; }
\end{aligned}
$$

- if $\sigma$ has length $n$ and $i$ is an element of $\omega$, then $z_{\sigma-\langle i+1\rangle}^{\bar{x}}$ is the union of $z_{\sigma}^{\bar{x}}$ with the singleton containing the $i$ th member of $x_{n}$ greater than all elements of $z_{\sigma}^{\bar{x}}$, where the least member is taken to be the 0th member.

Let $\pi_{\bar{x}}$ be the continuous embedding of the Baire space into $\mathcal{P}(\omega)$ induced by the assignment $\sigma \mapsto z_{\sigma}^{\bar{x}}$. Comeagerly many members of the Baire space map to infinite sets, and all of these are one-point selectors of $\left\langle x_{n}: n \in \omega\right\rangle$.

Remark 2.1. Given $\bar{x}$ as above, and $\sigma_{0}, \sigma_{1} \in \omega^{<\omega}$, there exist $\tau_{0}, \tau_{1} \in \omega^{<\omega}$ such that $\sigma_{0}^{\Upsilon} \tau_{0}$ and $\sigma_{1}^{\Upsilon} \tau_{1}$ have the same length, and $z_{\sigma_{0}}^{\bar{x}} \tau_{0}$ and $z_{\sigma_{1}}^{\bar{x}} \tau_{1}$ have the same 
maximal element. Furthermore, given $\rho_{0}, \rho_{1} \in \omega^{<\omega}$ with the same length, if $k$ is the maximal element of both $z_{\rho_{0}}^{\bar{x}}$ and $z_{\rho_{1}}^{\bar{x}}$, then for all $w \in \omega^{\omega}$,

$$
\pi_{\bar{x}}\left(\rho_{0} w\right) \backslash k=\pi_{\bar{x}}\left(\rho_{0} w\right) \backslash k .
$$

These observations are used in the first paragraph of the proof of Theorem 3.2

Definition 2.2. Let $\bar{x}=\left\langle x_{n}: n \in \omega\right\rangle$ and $\bar{y}=\left\langle y_{n}: n \in \omega\right\rangle$ be $\subseteq$-decreasing sequences of infinite subsets of $\omega$. We say that $\bar{x}$ and $\bar{y}$ are eventually intertwining if there exist $n, m \in \omega$ such that for all $k \in \omega$,

$$
y_{m+k+1} \subseteq x_{n+k+1} \subseteq y_{m+k} \subseteq x_{n+k} .
$$

The family of embeddings $\pi_{\bar{x}}$ was defined in order to make the following lemma hold.

Lemma 2.3. Let $\bar{x}$ and $\bar{y}$ eventually be intertwining sequences of infinite subsets of $\omega$, and let $C$ be a comeager subset of the Baire space. Then there exist $a, b \in C$ such that $\pi_{\bar{x}}(a)=\pi_{\bar{y}}(b)$.

Proof. We recursively define $\sigma_{n}, \tau_{n}$ in $\omega^{<\omega}$ for $n \in \omega$ as follows, maintaining the following conditions for all $n \in \omega$ :

$$
\begin{aligned}
& \text { - } \sigma_{n} \subseteq \sigma_{n+1} ; \\
& \text { - } \tau_{n} \subseteq \tau_{n+1} ; \\
& \text { - } z_{\sigma_{n}}^{\bar{x}}=z_{\tau_{n}}^{\bar{y}} .
\end{aligned}
$$

Let $n_{0}, m_{0} \in \omega$ witness that $\bar{x}$ and $\bar{y}$ are eventually intertwining. Let $\sigma_{0}$ consist of $n_{0}$ many 0's, and let $\tau_{0}$ consist of $m_{0}$ many 0's. Suppose that $n \in \omega$ and $\sigma_{n}$ and $\tau_{n}$ have been chosen. We need to see that, given a dense set $D \subseteq \omega^{<\omega}$, we can choose $\sigma_{n+1}$ and $\tau_{n+1}$ as above so that $\sigma_{n+1} \in D$ (we need to do this also for the $\tau_{n}$ 's, but the argument is symmetric). If necessary, we first extend $\sigma_{n}$ with 0 's to a sequence $\sigma_{n}^{\prime}$ of length at least $\left|\tau_{n}\right|+\left(n_{0}-m_{0}+1\right)$. Now let $\sigma_{n+1}$ be any extension of $\sigma_{n}^{\prime}$ in $D$. Suppose that $\left\langle i_{0}, \ldots, i_{p}\right\rangle$ is the sequence added to $\sigma_{n}^{\prime}$ to make $\sigma_{n+1}$. We can add a sequence $\left\langle j_{0}, \ldots, j_{p}\right\rangle$ to $\tau_{n}$ to make $\tau_{n+1}$, in such a way that for all $q \leq p$,

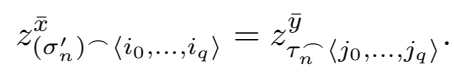

For each $q \leq p$, let $j_{q}=0$ if and only if $i_{q}=0$. Given $q \leq p$ such that $i_{q}>0$, the maximal element of $z_{\left(\sigma_{n}^{\prime}\right)-\left\langle i_{0}, \ldots, i_{q}\right\rangle}$ (call it $k$ ) is in $x_{\left|\sigma_{n}^{\prime}\right|+q}$, and therefore in $y_{\left|\tau_{n}\right|+q}$. It follows then that $j_{q}$ can be chosen as desired, so that $k$ is the $\left(j_{q}-1\right)$ th member of $y_{\left|\tau_{n}\right|+q}$ greater than all elements of $z_{\left(\sigma_{n}^{\prime}\right)-\left\langle i_{0}, \ldots, i_{q-1}\right\rangle}^{\bar{x}}$.

\section{EXTENDING A FUnCTION ON AN IDEAL}

Definition 3.1. Let $I$ be an ideal on $\omega$ containing Fin, and let $\mathcal{J}$ be a set of ideals on $\omega$. We say that the members of $\mathcal{J}$ are pairwise $I$-orthogonal if $x \cap y \in I$ whenever $J_{0}$ and $J_{1}$ are distinct elements of $\mathcal{J}$, and $x$ and $y$ are elements of $J_{0}$ and $J_{1}$ respectively. We let $\mathrm{FCI}(I, \mathcal{J})$ (the finite covering ideal) denote the ideal generated by $I \cup \cup \mathcal{J}$. For each $x \in \operatorname{FCI}(I, \mathcal{J}) \backslash I$, the set of $J \in \mathcal{J}$ such that $x \cap y \in I^{+}$for some $y \in J$ is finite and nonempty. We call this set $C(I, \mathcal{J}, x)$ below.

The following is our main result. In Theorems 3.2 and 3.3 , the formula defining the specified operation can be extracted from the proof, but we leave this to the interested reader. 
Theorem 3.2. Suppose that $\mathrm{AD}_{\mathbb{R}}$ holds. There exists a definable operation which takes in a nonempty countable set $X$, a tall ideal $I$ on $\omega$ containing Fin and a function

$$
f:(I \backslash \text { Fin }) \rightarrow\left([X]^{<\omega} \backslash\{\emptyset\}\right)
$$

which is invariant under finite changes, and returns a nonempty set $X^{\prime} \subseteq[X]^{<\omega}$ and a collection of pairwise $I$-orthogonal ideals $\left\{J_{a}: a \in X^{\prime}\right\}$ such that each $J_{a}$ properly contains $I$ and every I-positive set contains an I-positive set in some $J_{a}$.

Proof. By Corollary 1.2, Noselector has a winning strategy $\Gamma$ in the game $G^{\prime}(I)$. Suppose now that $\bar{x}$ is the sequence of moves played by Noselector in some run of $G^{\prime}(I)$ which has been won by Noselector. The function $f \circ \pi_{\bar{x}}$ maps a comeager subset of the Baire space into a countable set. Since all sets of reals have the Baire property, and $f$ is invariant under finite changes, $f \circ \pi_{\bar{x}}$ is constant on a comeager set (not merely a relatively comeager set; see Remark 2.1). Let $v_{\bar{x}}$ be this constant value.

Noselector can win the modified game in which he must declare with his first move the value $v_{\bar{x}}$ corresponding to the eventual run of the game. To see this, note that otherwise there exist a first move $w$ for Selector and a sequence of $G^{\prime}(I)$ strategies $\Sigma_{a}$ for Selector, for each nonempty $a \in[X]^{<\omega}$, each guaranteeing that either Selector wins the game or that the value $v_{\bar{x}}$ will not be $a$. Fixing some enumeration $\left\langle a_{i}: i \in \omega\right\rangle$ of $[X]^{<\omega} \backslash\{\emptyset\}$, consider the sequence that blends each $\Sigma_{a_{i}}$ against $\Gamma$, i.e.,

$$
\begin{aligned}
& \text { - } x_{0}=\Gamma(\langle w\rangle) \\
& \text { - } y_{0}^{0}=\Sigma_{a_{0}}\left(\left\langle x_{0}\right\rangle\right) \\
& \text { - } x_{1}=\Gamma\left(\left\langle w, x_{0}, y_{0}^{0}\right\rangle\right) \\
& \text { - } y_{1}^{0}=\Sigma_{a_{0}}\left(\left\langle x_{0}, y_{0}^{0}, x_{1}\right\rangle\right) \\
& \text { - } y_{0}^{1}=\Sigma_{a_{1}}\left(\left\langle y_{1}^{0}\right\rangle\right) \\
& \text { - } x_{2}=\Gamma\left(\left\langle w, x_{0}, y_{0}^{0}, x_{1}, y_{0}^{1}\right\rangle\right) \\
& \text { - } y_{2}^{0}=\Sigma_{a_{0}}\left(\left\langle x_{0}, y_{0}^{0}, x_{1}, y_{1}^{0}, x_{2}\right\rangle\right) \\
& \text { - } y_{1}^{1}=\Sigma_{a_{1}}\left(\left\langle y_{1}^{0}, y_{0}^{1}, y_{2}^{0}\right\rangle\right) \\
& \text { - } y_{0}^{2}=\Sigma_{a_{2}}\left(\left\langle y_{1}^{1}\right\rangle\right) \\
& \text { - } x_{3}=\Gamma\left(\left\langle w, x_{0}, y_{0}^{0}, x_{1}, y_{0}^{1}, x_{2}, y_{0}^{2}\right\rangle\right) \\
& \text { - } y_{3}^{0}=\Sigma_{a_{0}}\left(\left\langle x_{0}, y_{0}^{0}, x_{1}, y_{1}^{0}, x_{2}, y_{2}^{0}, x_{3}\right\rangle\right) \\
& \text { - } y_{2}^{1}=\Sigma_{a_{1}}\left(\left\langle y_{1}^{0}, y_{0}^{1}, y_{2}^{0}, y_{1}^{1}, y_{3}^{0}\right\rangle\right) \\
& \text { - } y_{1}^{2}=\Sigma_{a_{2}}\left(\left\langle y_{1}^{1}, y_{0}^{2}, y_{2}^{1}\right\rangle\right) \\
& \text { - } y_{0}^{3}=\Sigma_{a_{3}}\left(\left\langle y_{1}^{2}\right\rangle\right) \\
& \text { - } x_{4}=\Gamma\left(\left\langle w, x_{0}, y_{0}^{0}, x_{1}, y_{0}^{1}, x_{2}, y_{0}^{2}, x_{3}, y_{0}^{3}\right\rangle\right) \\
& \text { S }
\end{aligned}
$$

and so on. Then the sequence $\bar{x}=\left\langle x_{n}: n \in \omega\right\rangle$ is the sequence of moves for Noselector in a run of $G^{\prime}(I)$ according to $\Gamma$, and thus winning for Noselector. Letting $i$ be such that $a_{i}=v_{\bar{x}}$, let $\bar{y}$ be $\bar{x}$ in the case where $i=0$ and

$$
\left\langle y_{n+1}^{i-1}: n \in \omega\right\rangle
$$

otherwise. Then $\bar{y}$ is the sequence of moves for Noselector in a run of $G^{\prime}(I)$ according to $\Sigma_{a_{i}}$. However, $\bar{x}$ and $\bar{y}$ are eventually intertwining, which by Lemma 2.3 implies that the comeager constant values for these two sequences are the same, giving a contradiction.

Let $Z$ be the set of pairs $(x, a) \in I^{+} \times\left([X]^{<\omega} \backslash\{\emptyset\}\right)$ such that Noselector has a winning strategy in the modified version of $G^{\prime}(I)$ where Selector must play $\omega$ with his first move and Noselector must play $x$ and declare $a$ as the eventual constant 
value for $\bar{x}$. Let $X^{\prime}$ be the set of $a$ for which there exists an $x$ with $(x, a) \in Z$, and for each $a \in X^{\prime}$, let $J_{a}$ be the union of $I$ with the set of $x$ such that $(x, a) \in Z$. Then each $J_{a}$ is an ideal, and the previous paragraph shows that every $I$-positive set contains an $I$-positive member of some $J_{a}$.

Another strategy-blending argument shows that the ideals $J_{a}$ are pairwise $I$ orthogonal. Fix $(x, a)$ and $\left(x^{\prime}, a^{\prime}\right)$ in $Z$ as witnessed (respectively) by strategies $\Delta$ and $\Delta^{\prime}$, and suppose that $x \cap x^{\prime} \in I^{+}$.

Consider the sequences defined by

$$
\begin{aligned}
& \text { - } x_{0}=x \\
& \text { - } x_{0}^{\prime}=x^{\prime} \\
& \text { - } x_{1}=\Delta\left(\left\langle\omega, x, x \cap x^{\prime}\right\rangle\right) \\
& \text { - } x_{1}^{\prime}=\Delta^{\prime}\left(\left\langle\omega, x^{\prime}, x_{1}\right\rangle\right) \\
& \text { - } x_{2}=\Delta\left(\left\langle\omega, x, x \cap x^{\prime}, x_{1}, x_{1}^{\prime}\right\rangle\right) \\
& \text { - } x_{2}^{\prime}=\Delta^{\prime}\left(\left\langle\omega, x^{\prime}, x_{1}, x_{1}^{\prime}, x_{2}\right\rangle\right) \\
& \text { - } x_{3}=\Delta\left(\left\langle\omega, x, x \cap x^{\prime}, x_{1}, x_{1}^{\prime}, x_{2}, x_{2}^{\prime}\right\rangle\right) \\
& \text { - } x_{3}^{\prime}=\Delta^{\prime}\left(\left\langle\omega, x^{\prime}, x_{1}, x_{1}^{\prime}, x_{2}, x_{2}^{\prime}, x_{3}\right\rangle\right)
\end{aligned}
$$

and so on. Then $\left\langle x_{n}: n \in \omega\right\rangle$ lists Noselector's moves for a run of $G^{\prime}(I)$ according to $\Delta,\left\langle x_{n}^{\prime}: n \in \omega\right\rangle$ lists Noselector's moves for a run of $G^{\prime}(I)$ according to $\Delta^{\prime}$, and the two sequences are eventually intertwining. Applying Lemma 2.3 again then completes the proof.

Given $I, X, f$, and $\mathcal{J}=\left\{J_{a}: a \in X^{\prime}\right\}$ as in Theorem 3.2, $f$ can be extended, using the map $x \mapsto C(I, \mathcal{J}, x)$, to a function on $\operatorname{FCI}(I, \mathcal{J})$ which is invariant under finite changes and has codomain $[X]^{<\omega}$. Iterating this fact, possibly transfinitely, one must eventually add $\omega$ to the domain of the extended function. This gives the following result.

Theorem 3.3. Suppose that $\mathrm{AD}_{\mathbb{R}}$ holds. There is a definable operation which takes in a function of the form $f:(I \backslash$ Fin $) \rightarrow\left([X]^{<\omega} \backslash\{\emptyset\}\right)$, where $I$ is a tall ideal on $\omega$ containing Fin, $X$ is a countable set and $f$ is invariant under finite changes, and returns a function $f^{\prime}:(\mathcal{P}(\omega) \backslash$ Fin $) \rightarrow\left([X]^{<\omega} \backslash\{\emptyset\}\right)$, also invariant under finite changes, such that $f^{\prime}(x)=f(x)$ for all $x \in I \backslash$ Fin.

The following corollary was our motivation for proving this theorem.

Corollary 3.4. Suppose that $\mathrm{AD}_{\mathbb{R}}$ holds. Let $Y$ be a set, and let $G$ be a function with domain $Y$ such that $G(y)$ is countable for all $y \in Y$. Suppose that there exists a $\mathcal{P}(\omega)$ /Fin-name for a function on $Y$ which picks a nonempty finite subset of $G(y)$ for each $y \in Y$. Then there exists already a function on $Y$ which picks a nonempty finite subset of $G(y)$ for each $y \in Y$.

Proof. We show how to pick a finite subset of $G(y)$ for each $y \in Y$. Fix $y \in Y$. For each nonempty finite $a \subseteq G(y)$, let $J_{a}$ be the union of Fin with the set of infinite $x \subseteq \omega$ forcing the value of $\tau$ at $y$ to be $a$. Let $\mathcal{J}$ be the collection of nonempty sets of the form $J_{a}$. Then $\mathcal{J}$ is a collection of pairwise orthogonal ideals, and FCI(Fin, $\mathcal{J}$ ) is a tall ideal. Define

$$
f:(\text { FCI }(\text { Fin, } \mathcal{J}) \backslash \text { Fin }) \rightarrow\left([G(y)]^{<\omega} \backslash\{\emptyset\}\right)
$$

by setting $f(x)=\bigcup\left\{a \mid J_{a} \in C(I, \mathcal{J}, x)\right\}$. Then apply Theorem 3.3 with $f$, and let $f^{\prime}(\omega)$ be the chosen set. 
As a consequence, forcing with $\mathcal{P}(\omega) /$ Fin over a model of $\mathrm{AD}_{\mathbb{R}}$ does not add an $E_{0}$-selector. Furthermore, for any pair of equivalence relations $E, F$ in a model $M$ of $\mathrm{AD}_{\mathbb{R}}$ for which every $E$-class is made up of countably many $F$-classes, if in a $\mathcal{P}(\omega) /$ Fin extension of $M$ there is a function which picks a finite set of $F$-subclasses for each $E$-class, then such a function exists in $M$.

The argument for Corollary 3.4 also shows the following.

Corollary 3.5. Suppose that $\mathrm{AD}_{\mathbb{R}}$ holds. Then there is a function which takes in a countable family of orthogonal ideals whose union is dense in $\mathcal{P}(\omega) /$ Fin and returns a finite subfamily.

Prikry [5] proved from $\mathrm{AD}_{\mathbb{R}}$ that every set of reals is Ramsey. Through work of Martin, Steel and Woodin this assumption was improved to $\mathrm{AD}^{+}$(see [1]). The following question asks for an analogous improvement for Theorem 3.2

Question 3.6. Can the assumption of $\mathrm{AD}_{\mathbb{R}}$ in Theorem 3.2 be replaced by $\mathrm{AD}^{+}$?

Another natural question is the following.

Question 3.7. Are there uncountable sets $X$ for which Theorem 3.3 holds?

\section{EXAMPLES}

By convention, $\Theta$ denotes the least ordinal which is not a surjective image of $\mathcal{P}(\omega)$. The iterated application of Theorem 3.2 in the proof of Theorem 3.3 clearly cannot run for $\Theta$ steps. Let us say that a function $f$ as in Theorem 3.2 has rank $\alpha$ if it takes exactly $\alpha$ many iterations of Theorem 3.2 to add $\omega$ to the domain of the function $f^{\prime}$ as in Theorem 3.3. We show in this section that, assuming $\mathrm{AD}_{\mathbb{R}}$, there exist functions of rank $\alpha$ for cofinally many $\alpha<\Theta$. Our first example below illustrates a function of rank 1 . The second shows how to combine a collection of functions, indexed by an almost disjoint family, to produce a function whose rank is greater than all the members of the collection.

Example 4.1. Suppose that $\left\{a_{i}: 1 \leq i<\omega\right\}$ is a partition of $\omega$ into infinite sets. For each positive $i \in \omega$, let $J_{i}$ be the set of $x \subseteq \omega$ for which $x \backslash a_{i}$ is finite. Let $J_{0}$ be the set of $x \subseteq \omega$ having finite intersection with each $a_{i}$, and let $\mathcal{J}=\left\{J_{i}: i \in \omega\right\}$. Define $f:(\mathrm{FCI}($ Fin, $\mathcal{J}) \backslash$ Fin $) \rightarrow\left([\mathcal{J}]^{<\omega} \backslash\{\emptyset\}\right)$ by letting $f(x)=C($ Fin, $\mathcal{J}, x)$. Then every position (in particular, $\omega$ ) in the game from the proof of Theorem 3.2 is a winning position for Noselector with value $\left\{J_{0}\right\}$, so $f$ extends to all of $\mathcal{P}(\omega) \backslash$ Fin in one step.

Example 4.2. Suppose that we are given a countable set $X$ (with $\emptyset \notin X$ ), a nonmaximal almost disjoint family $\mathcal{A}$ of infinite subsets of $\omega$ (so distinct members of $\mathcal{A}$ have finite intersection), and ideals $I_{a}$ (properly containing Fin) and functions $f_{a}:\left(I_{a} \backslash\right.$ Fin $) \rightarrow\left([X]^{<\omega} \backslash\{\emptyset\}\right)(a \in \mathcal{A})$ as in Theorem 3.2. Suppose that $\alpha_{a}(a \in \mathcal{A})$ are ordinals such that each $f_{a}$ takes $\alpha_{a}$ many applications of Theorem 3.2 to add $\omega$ to its domain. Let $J_{\emptyset}$ be $\mathcal{A}^{\perp}$, the set of $x \subseteq \omega$ having finite intersection with each $a \in \mathcal{A}$. For each $a \in \mathcal{A}$, let $\pi_{a}: \omega \rightarrow a$ be a bijection. For each $a \in \mathcal{A}$, let $J_{a}$ be the ideal generated by Fin $\cup\left\{\pi_{a}[x]: x \in I_{a}\right\}$, and define $f_{a}^{\prime}:\left(J_{a} \backslash\right.$ Fin $) \rightarrow\left([X]^{<\omega} \backslash\{\emptyset\}\right)$ by setting $f_{a}^{\prime}(x)=f_{a}\left(\pi_{a}^{-1}[x \cap a]\right)$. Let $\mathcal{J}=\left\{J_{\emptyset}\right\} \cup\left\{J_{a}: a \in \mathcal{A}\right\}$, and define 
$f:(\mathrm{FCI}(\mathrm{Fin}, \mathcal{J}) \backslash \mathrm{Fin}) \rightarrow\left([\{\emptyset\} \cup X]^{<\omega} \backslash\{\emptyset\}\right)$ by letting

- $\emptyset \in f(x)$ if and only if $J_{0} \in C($ Fin, $\mathcal{J}, x)$,

- for all $z \in X, z \in f(x)$ if and only if there exists $a \in \mathcal{A}$ such that $J_{a} \in$ $C($ Fin, $\mathcal{J}, x)$ and $z \in f_{a}^{\prime}(x)$.

The iterated application of Theorem 3.2 for $f$ adds subsets of each $a \in \mathcal{A}$ exactly when the corresponding process for $f_{a}$ adds the $\pi_{a}$-preimages of these sets. It follows that it takes $\alpha_{a}$ many iterations of Theorem 3.2 to add each $a \in \mathcal{A}$ to the extended domain of $f$, and more than $\sup \left\{\alpha_{a}: a \in \mathcal{A}\right\}$ many iterations to add $\omega$.

Fixing a bijection $g$ : Fin $\rightarrow \omega$, the function which sends $a \in \mathcal{P}(\omega)$ to

$$
\{g(a \cap n): n \in \omega\}
$$

is Borel and sends distinct infinite sets to infinite sets with finite intersection. It follows then (under ZF) that for each $\alpha<\Theta$ there is a surjection $\rho: \mathcal{A} \rightarrow \alpha$, for some almost disjoint family $\mathcal{A}$. Starting with a function as in Example 4.1, one can iteratively apply the construction from Example 4.2 filling in functions of rank $\beta$ for the indices in $\rho^{-1}[\{\beta\}]$, to produce a function of rank at least $\alpha$.

\section{Counterexamples}

The Axiom of Real Determinacy implies that every set of reals has the property of Baire, and this precludes a number of variations of the result proved in this paper. In this section we show that "finite subset of $G(y)$ " cannot be replaced with "element of $[G(y)]^{\leq n} \backslash\{\emptyset\}$ " in Corollary 3.4 for any positive $n \in \omega$, even if $\tau$ is a name for a function picking a single element from each $G(y)$. Similarly, "finite subfamily" cannot be replaced with "nonempty subfamily of size at most $n$ " in Corollary 3.5 .

Example 5.1. Suppose that every set of reals has the property of Baire. Then there is no function $F: \mathcal{P}(\omega) \rightarrow 2$ with the property that for all subsets $x, y$ of $\omega, F(x) \neq F(y)$ if $x \triangle(\omega \backslash y)$ is finite (i.e., no function which chooses between complementary pairs of $E_{0}$-degrees). This follows from the fact that $F$ would be invariant under finite changes, so one of $F^{-1}[\{0\}]$ and $F^{-1}[\{1\}]$ would have to be comeager, but any comeager subset of $\mathcal{P}(\omega)$ contains a pair of complements. A similar argument shows (under the same assumption) that, for any $n \in \omega$, there is no function which takes in a collection $\left\{\left[a_{i}\right]_{E_{0}}: i<n\right\}$, for $\left\{a_{i}: i<n\right\}$ a partition of $\omega$, and returns a nonempty proper subset. If there were such a function, there would be $j, k<n$ such that for comeagerly many partitions $\left\langle a_{i}: i<n\right\rangle$ of $\omega,\left[a_{j}\right]_{E_{0}}$ would be in the chosen subset, and $\left[a_{k}\right]_{E_{0}}$ would not. However, comeagerly many members of this set would have the property that switching the values of $a_{j}$ and $a_{k}$ would give another member of the set, giving a contradiction.

To see this example in terms of Theorem 3.3, notice that a nonprincipal ultrafilter on $\omega$ induces a choice of one element from each set of the form

$$
\left\{\left[a_{i}\right]_{E_{0}}: i \leq n\right\},
$$

for $\left\{a_{i}: i \leq n\right\}$ a partition of $\omega$ into infinite sets. The previous paragraph shows that there is no function in a model of $\mathrm{AD}_{\mathbb{R}}$ picking a proper subfamily from each such family.

The previous example does not give a counterexample corresponding to Theorem 3.3. as the corresponding ideal FCI $(\mathrm{Fin}, \mathcal{J})$ would be all of $\mathcal{P}(\omega)$. We remedy this by 
showing how to replace each set of the form $[a]_{E_{0}}$ with an infinite maximal antichain in $\mathcal{P}(a) / E_{0}$. Once we have this, we can replace each set $\left\{\left[a_{i}\right]_{E_{0}}: i \leq n\right\}$ as above with the union of the corresponding antichains. Then as before, no function in the ground model can pick a subfamily of size at most $n$ from each such union. The corresponding function $f$ as in Theorem 3.3 has the form $x \mapsto C($ Fin, $\mathcal{J}, x)$ for $x \in \mathrm{FCI}(\mathrm{Fin}, \mathcal{J})$ and $\mathcal{J}$ the collection of ideals derived from this antichain.

The only missing ingredient is a method for taking a set of the form $[a]_{E_{0}}$, for $a \subseteq \omega$ infinite, and returning a set of the form $[b]_{E_{0}}$, for some infinite $b \subseteq a$ for which $a \backslash b$ is infinite. The following observation does what we need.

Lemma 5.2. There is a Borel function $g$ that assigns each to infinite $a \subseteq \omega$ an infinite $g(a) \subseteq$ a for which $a \backslash g(a)$ is infinite, in such a way that $a \triangle b \in$ Fin implies that $g(a) \triangle g(b) \in$ Fin.

Proof. For each $n \in \omega$, let $F_{n}$ be the equivalence relation on $\mathcal{P}(\omega)$ defined by $a F_{n} b \Leftrightarrow(a \backslash n)=(b \backslash n)$. Then define functions $S_{n}(n \in \omega)$ on $\mathcal{P}(\omega)$ by

- $S_{0}(a)$ is the second element of $a$,

- $S_{n+1}(a)$ is the second element of $\bigcap[a]_{F_{n+1}} \backslash\left(\sup \left\{S_{n}(b)+1: b \in[a]_{F_{n+1}}\right\}\right)$.

Then for each infinite $a \subseteq \omega$ and each $n \in \omega, S_{n}(a) \geq 2 n+1$, and $\bigcap[a]_{F_{n+1}}$ can equivalently be replaced with $a$ in the definition of $S_{n+1}(a)$. It follows that the function $g(a)=\left\{S_{n}(a): n \in \omega\right\}$ is as desired.

\section{REFERENCES}

[1] Andrés Eduardo Caicedo and Richard Ketchersid, A trichotomy theorem in natural models of $\mathrm{AD}^{+}$, Set theory and its applications, Contemp. Math., vol. 533, Amer. Math. Soc., Providence, RI, 2011, pp. 227-258, DOI 10.1090/conm/533/10510. MR2777751 (2012i:03146)

[2] C. A. Di Prisco and S. Todorcevic, Souslin partitions of products of finite sets, Adv. Math. 176 (2003), no. 1, 145-173, DOI 10.1016/S0001-8708(02)00064-6. MR 1978344 (2004f:03083)

[3] P. Larson, D. Raghavan, Real games and strategically selective coideals, in preparation.

[4] A. R. D. Mathias, Happy families, Ann. Math. Logic 12 (1977), no. 1, 59-111. MR0491197 (58 \#10462)

[5] Karel Prikry, Determinateness and partitions, Proc. Amer. Math. Soc. 54 (1976), 303-306. MR0453540 (56 \#11802)

[6] Saharon Shelah and Jindřich Zapletal, Ramsey theorems for product of finite sets with submeasures, Combinatorica 31 (2011), no. 2, 225-244, DOI 10.1007/s00493-011-2677-5. MR2848252 (2012f:05312)

Department of Mathematics, Miami University, Oxford, Ohio 45056

E-mail address: larsonpb@miamioh.edu 